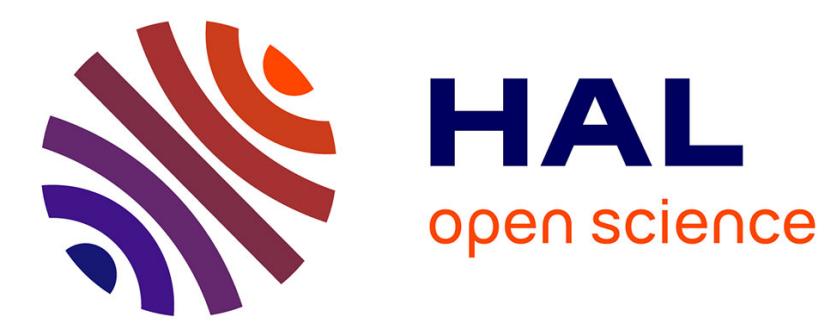

\title{
Generic properties of closed orbits of Hamiltonian flows from Mañé's viewpoint
}

Ludovic Rifford, Rafael Ruggiero

\section{To cite this version:}

Ludovic Rifford, Rafael Ruggiero. Generic properties of closed orbits of Hamiltonian flows from Mañé's viewpoint. International Mathematics Research Notices, 2011, 2012, pp.5246. hal-00923322

\section{HAL Id: hal-00923322 \\ https://hal.science/hal-00923322}

Submitted on 2 Jan 2014

HAL is a multi-disciplinary open access archive for the deposit and dissemination of scientific research documents, whether they are published or not. The documents may come from teaching and research institutions in France or abroad, or from public or private research centers.
L'archive ouverte pluridisciplinaire HAL, est destinée au dépôt et à la diffusion de documents scientifiques de niveau recherche, publiés ou non, émanant des établissements d'enseignement et de recherche français ou étrangers, des laboratoires publics ou privés. 


\title{
GENERIC PROPERTIES OF CLOSED ORBITS OF HAMILTONIAN FLOWS FROM MAÑÉ'S VIEWPOINT
}

\author{
L. RIFFORD AND R. RUGGIERO
}

\begin{abstract}
We show the genericity from the viewpoint of Mañé of generic properties (as symplectic linear maps) of the differential of Poincaré maps of periodic orbits of Hamiltonians. Combining this result and the work of Oliveira [19] we get a Kupka-Smale type theorem " à la Mañé" for regular energy levels of Tonelli Hamiltonians in compact manifolds. Our proof relies on techniques from geometric control theory.
\end{abstract}

\section{INTRODUCTION}

The remarkable work of Ricardo Mañé about Aubry-Mather theory for $C^{\infty}$, convex, superlinear Lagrangians $L: T M \longrightarrow \mathbb{R}$, or Tonelli Lagrangians, gives us the starting point of a program to understand genericity of Lagrangians and Hamiltonians from a special point of view. Genericity for a certain system property means dense or Baire genericity of the family of systems enjoying such property in an appropriate topological space.

Definition 1.1. Let $M$ be a $C^{\infty}$ compact manifold. We say that a property $P$ of $C^{k}$ Tonelli Lagrangians in $M, L: T M \longrightarrow \mathbb{R}$, is Mañé $C^{r}$ generic, for $r \leq k$ if there exists a $C^{r}$ generic set of $C^{k}$ functions $U: M \longrightarrow \mathbb{R}$ such that the property $P$ holds for each Lagrangian of the form $L_{U}(p, v)=L(p, v)-U(p)$. We shall say that $P$ is Mañé $C^{r}$ generic. This definition proceeds for Hamiltonians $H$ replacing $L_{U}=L-U$ by $H_{U}=H+U$. Analogously, we define the Mañé $C^{\infty}$ genericity of a property $P$ by replacing $C^{k}, C^{r}$ by $C^{\infty}$ in the above statement.

The family $L_{U}(p, v)=L(p, v)+U(p)$ of Lagrangians is a very natural family of conservative systems: when $L(p, v)=\frac{1}{2} g_{p}(v, v)$ is a Riemannian metric, the above Lagrangians are just mechanical Lagrangians. The study of the genericity from Mañé's view point amounts to study perturbations of Lagrangians or Hamiltonians obtained just by adding a small scalar function to the Lagrangian, or a small potential in the terminology of classical mechanics. In the Riemannian case, such perturbations correspond to conformal perturbations of the Riemannian metric. 
The simplicity of the idea of perturbing just by adding small potentials, and the deep preliminary results obtained by Mañé about generic properties of AubryMather sets (invariant objects of Lagrangian systems with action minimizing properties) had a strong appeal to researchers in classical mechanics and dynamical systems. Since Mañé's initial paper [15], a great deal of work has been devoted to understand generic properties of systems from this point of view (see Massart [16], Contreras [6], Contreras and Iturriaga [7], Contreras and Paternain [8], Oliveira [19], Ruggiero [23], etc..), specially to solve his famous conjecture about the generic uniqueness of the Aubry-Mather set in a specified homology class (see Bernard and Contreras [5] or Figalli and Rifford [11, 12]). However, the apparent simplicity of this sort of perturbations is in contrast with the highly technical difficulties arising from the fact that this family of perturbations is a very restricted one. As we mentioned before, in the case of Riemannian metrics Mañé's genericity is equivalent to attain generic properties of geodesic flows just by considering conformal perturbations of metrics.

This point of view of genericity leads to many interesting questions concerning the usual theory of generic dynamical systems: Is the $C^{k}$ genericity theory of Lagrangians (Hamiltonians) equivalent to Mañé $C^{k}$ genericity? Does the celebrated $C^{1}$ closing lemma for flows hold for perturbations of Lagrangians by potentials? Remark that the $C^{1}$ closing lemma for geodesic flows is not known (see Rifford [20] for a proof of the $C^{0}$ closing lemma for geodesic flows just by adding small $C^{1}$ potentials to the metric). Classical results of generic properties of the Poincaré map of closed orbits are due to Abrahams [1], Anosov [3] (the so-called bumpy metric theorem); and by Klingenberg-Takens [13] for Hamiltonians. Robinson [21, 22] showed the KupkaSmale theorem for Hamiltonian flows; and Devaney [10] showed the Kupka-Smale theorem for reversible flows, namely, flows commuting with an involution defined in the manifold (like Hamiltonian flows of mechanical Lagrangians). None of the proofs of these results proceeds when perturbing the Hamiltonian by potentials.

In this article we consider the Kupka-Smale theorem for Hamiltonian flows from Mañé's viewpoint. The Kupka-Smale theorem, one of the first important results about generic properties of dynamical systems, asserts that the set of $C^{k}$ diffeomorphisms and flows in a compact manifold satisfying the following two properties:

(1) All closed orbits are hyperbolic.

(2) Heteroclinic orbits are transversal, i.e., the intersection between stable and unstable manifolds of closed hyperbolic orbits are transversal,

is $C^{r}$ dense for every $r \leq k$ and $C^{1}$ residual in the set of diffeomorphisms. For conservative dynamical systems it is well known that the genericity of closed hyperbolic 
orbits is not true due to the symplectic properties of the differential of the Poincaré transform. So an appropriate version of the Kupka-Smale theorem for Hamiltonians in our setting would be that $C^{k}$ systems, $k \geq 2$, satisfying the following properties:

(1) Each closed orbit is either hyperbolic or no eigenvalue of the Poincaré transform of any closed orbit is a root of unity.

(2) Heteroclinic orbits are transversal, i.e., the intersection between stable and unstable manifolds of closed hyperbolic orbits are transversal,

are $C^{r}$ generic for every $r \leq k$ from Mañé's viewpoint ( $k$ might be $\infty$ ).

The problem has been already considered by Oliveira [19] who shows the KupkaSmale theorem for energy levels of Hamiltonians in compact surfaces from the point of view of Mañé, (assuming that genericity for potentials means density). Actually, in [19] the Mañé $C^{\infty}$ genericity of the transversal intersections of stable and unstable submanifolds of closed orbits is proved for n-dimensional compact manifolds So item (2) holds in fact for any dimension.

The proof of item (1) in [19] has two steps as in the usual proof of Kupka-Smale theorem for diffeomorphisms and flows. First of all, it is proved a transversality result, which holds in any dimension, called in [19] the reduction lemma: given an energy level, and given $T>0$, the set of Hamiltonians whose Hamiltonian flows in the level have a finite number of closed orbits of period at most $T$ is Mañé $C^{\infty}$ generic. The reduction lemma implies that item (1) follows from the proof of the genericity of the required spectral properties in each single orbit. This last step of the Kupka-Smale theorem is showed in [19] in dimension 2 (see Theorem 18 in [19]). Our contribution to the problem is the proof of the last step in any dimension, concluding in this way the proof of the Kupka-Smale theorem from the point of view of Mañé.

Theorem 1.2. Let $M$ be a $C^{\infty}$ compact manifold $M$ and $H: T^{*} M \longrightarrow \mathbb{R}$ be a $C^{k}$ Tonelli Hamiltonian, $k \geq 2$. Given a regular energy level $H^{-1}(E)$ of $H$, a closed orbit $\theta \in H^{-1}(E)$ and $\epsilon>0$, there is a $C^{\infty}$ function $U: M \longrightarrow \mathbb{R}$ whose $C^{k}$ norm is at most $\epsilon$ such that the Hamiltonian $H_{U}(p, q)=H(p, q)+U(p)$ satisfies the following properties:

(1) $\theta$ is a closed orbit of the Hamiltonian flow of $H_{U}$ with the same period and energy $E$.

(2) The differential of the Poincaré map of the orbit of $\theta$ with respect to the Hamiltonian flow of $H_{U}$ is generic in the set of symplectic linear transformations: each eigenvalue of the Poincaré map is either unitary and not a root of 1 , or is not unitary. 
The usual approach to tackle Theorem 1.2 is essentially the following. Let us consider perturbations of the Hamiltonian preserving a small subset $\gamma([a, b])$ given by the canonical projection of a closed orbit $\phi_{t}(\theta)=(\gamma(t), \beta(t))$ of the Hamiltonian flow. Then it is not difficult to write the perturbed differential of the Poincaré map in terms of the differential of the initial Poincaré map. In appropriate coordinates, this is given in terms of the perturbation of a Jacobi equation associated to the Hamiltonian flow. The differential of a perturbed Poincaré map satisfies a first order ordinary linear system whose homogeneous part is the differential of the unperturbed one. So the goal is to show that the collection of perturbed fundamental solutions of this family of systems whose initial conditions coincide with an unperturbed fundamental solution at $\gamma(a)$ attains an open set of symplectic matrices at $\gamma(b)$. This transversality statement can be proved in many ways, typically involving an integration of the perturbed system, a procedure posing highly technical problems.

Our strategy is to avoid integration by using tools of geometric control theory: the above problem can be set as a controllability problem. Control theory ideas give rise to a much simpler, geometric proof of Theorem 1.2 compared with previous works in the literature about generic properties of Poincaré maps of closed orbits of Hamiltonians.

Acknowledgements: We would like to thank Professor Mário Jorge D. Carneiro from the Universidade Federal de Minas Gerais, and Professor Elismar Oliveira from the Universidade Federal de Rio Grande do Sul, for fruitful discussions about the subject.

\section{Preliminaries in CONTROL TheOrY}

Our aim here is to provide simple sufficient conditions for local controllability results. This kind of results could be developped for nonlinear control systems on smooth manifolds. For sake of simplicity, we restrict our attention here to the case of affine control systems on the set of (symplectic) matrices. We refer the interested reader to [9] for a further study in control theory.

Let us a consider a control system on $M_{2 m}(\mathbb{R})$ (with $m, k \geq 1$ ), of the form

$$
\dot{X}(t)=A(t) X(t)+\sum_{i=1}^{k} u_{i}(t) B_{i}(t) X(t), \quad \text { for a.e. } t
$$

where the state $X(t)$ belongs to $M_{2 m}(\mathbb{R})$, the control $u(t)$ belongs to $\mathbb{R}^{k}$, and $t \in$ $[0, T] \mapsto A(t), B_{1}(t), \ldots, B_{k}(t)$ are $k+1$ smooth maps valued in $M_{2 m}(\mathbb{R})$ (with 
$T>0)$. Given $\bar{X} \in M_{2 m}(\mathbb{R})$ and $\bar{u} \in L^{1}\left([0, T] ; \mathbb{R}^{k}\right)$, the Cauchy problem

(2) $\dot{X}(t)=A(t) X(t)+\sum_{i=1}^{k} \bar{u}_{i}(t) B_{i}(t) X(t), \quad$ for a.e. $\quad t, \quad X(0)=\bar{X}$,

possesses a unique maximal solution $X_{\bar{X}, \bar{u}}(\cdot)$ defined on a maximal interval of the form $\left[0, T_{\bar{X}, \bar{u}}\right)$ with $T_{\bar{X}, \bar{u}} \in[0, T]$. Given $\bar{X} \in M_{2 m}(\mathbb{R})$, we denote by $\mathcal{U}_{\bar{X}}$ the set of controls $u \in L^{1}\left([0, T] ; \mathbb{R}^{k}\right)$ such that $T_{\bar{X}, u}=T$. The set $\mathcal{U}_{\bar{X}}$ is an open (possibly empty) subset of $L^{1}\left([0, T] ; \mathbb{R}^{k}\right)$. The End-Point mapping associated with $\bar{X}$ in time $T$ (here we assume that the set is $\mathcal{U}_{\bar{X}}$ is nonempty) is defined as

$$
\begin{aligned}
E^{\bar{X}}: \mathcal{U}_{\bar{X}} & \longrightarrow M_{2 m}(\mathbb{R}) \\
u & \longmapsto X_{\bar{X}, u}(T) .
\end{aligned}
$$

It is a smooth mapping. Given $\bar{X} \in M_{2 m}(\mathbb{R}), \bar{u} \in \mathcal{U}_{\bar{X}}$, and setting $\bar{X}(\cdot):=X_{\bar{X}, u}(\cdot)$, the differential of $E^{\bar{X}}$ at $\bar{u}$ is given by the linear operator

$$
\begin{array}{cc}
d E^{\bar{X}}(\bar{u}): L^{1}\left([0, T] ; \mathbb{R}^{k}\right) & \longrightarrow M_{2 m}(\mathbb{R}) \\
v & \longmapsto Y(T),
\end{array}
$$

where $Y(\cdot)$ is the unique solution to the Cauchy problem

$$
\left\{\begin{array}{l}
\dot{Y}(t)=A(t) Y(t)+\sum_{i=1}^{k} v_{i}(t) B_{i}(t) \bar{X}(t) \text { for a.e. } t \in[0, T], \\
Y(0)=0 .
\end{array}\right.
$$

Note that if we denote by $S(\cdot)$ the solution to the Cauchy problem

$$
\left\{\begin{array}{l}
\dot{S}(t)=A(t) S(t) \\
S(0)=I_{n}
\end{array}\right.
$$

then there holds

$$
d E^{\bar{X}}(\bar{u})(v)=\sum_{i=1}^{k} S(T) \int_{0}^{T} v_{i}(t) S(t)^{-1} B_{i}(t) \bar{X}(t) d t,
$$

for every $v \in L^{1}\left([0, T] ; \mathbb{R}^{k}\right)$.

Let $\operatorname{Sp}(m)$ be the symplectic group in $M_{2 m}(\mathbb{R})(m \geq 1)$, that is the smooth submanifold of matrices $X \in M_{2 m}(\mathbb{R})$ satisfying

$$
X^{*} \mathbb{J} X=\mathbb{J} \quad \text { where } \mathbb{J}:=\left[\begin{array}{cc}
0 & I_{m} \\
-I_{m} & 0
\end{array}\right] .
$$


Denote by $\mathcal{S}(2 m)$ the set of symmetric matrices in $M_{2 m}(\mathbb{R})$. The tangent space to $\operatorname{Sp}(m)$ at the identity matrix is given by

$$
T_{I_{2 m}} \operatorname{Sp}(m)=\left\{Y \in M_{2 m}(\mathbb{R}) \mid \rrbracket Y \in \mathcal{S}(2 m)\right\} .
$$

Therefore, if there holds

$$
\mathbb{J} A(t), \mathbb{J} B_{1}(t), \ldots, \mathbb{J} B_{k}(t) \in \mathcal{S}(2 m) \quad \forall t \in[0, T],
$$

then $\operatorname{Sp}(m)$ is invariant with respect to (1), that is for every $\bar{X} \in M_{2 m}(\mathbb{R})$ and $\bar{u} \in \mathcal{U}_{\bar{X}}$,

$$
X_{\bar{X}, u}(t) \in \operatorname{Sp}(m) \quad \forall t \in[0, T] .
$$

In particular, this means that for every $\bar{X} \in \operatorname{Sp}(m)$, the End-Point mapping $E^{\bar{X}}$ is valued in $\operatorname{Sp}(m)$. Our aim is now to provide a sufficient condition of local controllability in $\operatorname{Sp}(m)$.

Proposition 2.1. Let $T>0$, and $t \in[0, T] \mapsto A(t), B_{1}(t), \ldots, B_{k}(t) \in M_{2 m}(\mathbb{R})$ be smooth mappings satisfying (6). Define the $k$ sequences of smooth mappings

$$
\left\{B_{1}^{j}\right\}, \ldots,\left\{B_{k}^{j}\right\}:[0, T] \rightarrow T_{I_{2 m}} S p(m)
$$

by

$$
\left\{\begin{array}{l}
B_{i}^{0}(t):=B_{i}(t) \\
B_{i}^{j}(t):=\dot{B}_{i}^{j-1}(t)+B_{i}^{j-1}(t) A(t)-A(t) B_{i}^{j-1}(t),
\end{array}\right.
$$

for every $t \in[0, T]$ and every $i \in\{1, \ldots, k\}$. Assume that there exists some $\bar{t} \in[0, T]$ such that

$$
\operatorname{Span}\left\{B_{i}^{j}(\bar{t}) \mid i \in\{1, \ldots, k\}, j \in \mathbb{N}\right\}=T_{I_{2 m}} S p(m) .
$$

Then for every $\bar{X} \in S p(m)$ such that $\bar{u} \equiv 0$ belongs to $\mathcal{U}_{\bar{X}} \subset L^{1}\left([0, T] ; \mathbb{R}^{k}\right)$ (associated with (1)), there holds

$$
d E^{\bar{X}}\left(L^{1}\left([0, T] ; \mathbb{R}^{k}\right)\right)=T_{\bar{X}(T)} S p(m),
$$

with $\bar{X}(T):=X_{\bar{X}, u}(T)$.

Proof. We need to show that $d E^{\bar{X}}: L^{1}\left([0, T] ; \mathbb{R}^{k}\right) \rightarrow T_{\bar{X}(T)} \operatorname{Sp}(m)$ is surjective. If not, there exists a nonzero matrix $Y \in M_{2 m}(\mathbb{R})$ such that

$$
\bar{X}(T)^{*} \mathbb{J} Y \in \mathcal{S}(2 m)
$$

and

$$
\operatorname{Tr}\left(Y^{*} d E^{\bar{X}}(\bar{u})(v)\right)=0 \quad v \in L^{1}\left([0, T] ; \mathbb{R}^{k}\right)
$$


By (5), this can be written as

$$
\sum_{i=1}^{k} \int_{0}^{T} v_{i}(t) \operatorname{Tr}\left(Y^{*} S(T) S(t)^{-1} B_{i}(t) \bar{X}(t)\right) d t=0 \quad \forall v \in L^{1}\left([0, T] ; \mathbb{R}^{k}\right) .
$$

Taking for every $i \in\{1, \ldots, k\}$,

$$
v_{i}(t):=\operatorname{Tr}\left(Y^{*} S(T) S(t)^{-1} B_{i}(t) \bar{X}(t)\right) \quad \forall t \in[0, T],
$$

we deduce that $\left(v(\cdot)\right.$ is smooth on $[0, T]$, so it belongs to $\left.L^{1}\left([0, T] ; \mathbb{R}^{k}\right)\right)$

$$
\sum_{i=1}^{k} \int_{0}^{T}\left[\operatorname{Tr}\left(Y^{*} S(T) S(t)^{-1} B_{i}(t) \bar{X}(t)\right)\right]^{2} d t=0
$$

which implies

$$
\operatorname{Tr}\left(Y^{*} S(T) S(t)^{-1} B_{i}(t) \bar{X}(t)\right)=0 \quad \forall t \in[0, T] .
$$

The above equality at $t=\bar{t}$ yields

$$
\operatorname{Tr}\left(Y^{*} S(T) S(\bar{t})^{-1} B_{i}^{0}(\bar{t}) \bar{X}(\bar{t})\right)=0 .
$$

Using that $\frac{d}{d t}\left(S(t)^{-1}\right)=-S(t)^{-1} A(t), \dot{\bar{X}}(t)=A(t) \bar{X}(t)$ and differentiating (10) at $t=\bar{t}$ gives

$$
\begin{aligned}
\operatorname{Tr}\left(Y^{*} S(T)\left(-S(\bar{t})^{-1} A(\bar{t})\right)\right. & \left.B_{i}^{0}(\bar{t}) \bar{X}(\bar{t})\right) \\
+ & \operatorname{Tr}\left(Y^{*} S(T) S(\bar{t})^{-1} \dot{B}_{i}^{0}(\bar{t}) \bar{X}(\bar{t})\right) \\
& +\operatorname{Tr}\left(Y^{*} S(T) S(\bar{t})^{-1} B_{i}^{0}(\bar{t})(A(\bar{t} \bar{X}(\bar{t})))=0 .\right.
\end{aligned}
$$

Which can be written as

$$
\operatorname{Tr}\left(Y^{*} S(T) S(\bar{t})^{-1} B_{i}^{1}(\bar{t}) \bar{X}(\bar{t})\right)=0 .
$$

Differentiating again and again yields that

$$
\operatorname{Tr}\left(Y^{*} S(T) S(\bar{t})^{-1} B_{i}^{j}(\bar{t}) \bar{X}(\bar{t})\right)=0 \quad \forall j \in \mathbb{N}, \forall i \in\{1, \ldots, k\} .
$$

Let us show that all the matrices $S(T) S(\bar{t})^{-1} B_{i}^{j}(\bar{t}) \bar{X}(\bar{t})$ belong to $T_{\bar{X}(T)} \operatorname{Sp}(m)$. For that we need to prove that for any $i \in\{1, \ldots, k\}$ and any $j \in \mathbb{N}$,

$$
\bar{X}(T)^{*} \mathbb{J}\left(S(T) S(\bar{t})^{-1} B_{i}^{j}(\bar{t}) \bar{X}(\bar{t})\right) \in \mathcal{S}(2 m) .
$$

Since $\bar{u} \equiv 0$, there holds

$$
\bar{X}(T)=S(T) \bar{X} \quad \text { and } \quad \bar{X}(\bar{t})=S(\bar{t}) \bar{X} .
$$


Then

$$
\begin{aligned}
\bar{X}(T)^{*} \mathbb{J}\left(S(T) S(\bar{t})^{-1} B_{i}^{j}(\bar{t}) \bar{X}(\bar{t})\right) & =\bar{X}(T)^{*} \mathbb{J} \bar{X}(T) \bar{X}^{-1} S(\bar{t})^{-1} B_{i}^{j}(\bar{t}) \bar{X}(\bar{t}) \\
& =\mathbb{J} \bar{X}^{-1} S(\bar{t})^{-1} B_{i}^{j}(\bar{t}) \bar{X}(\bar{t}) \\
& =\mathbb{J} \bar{X}(\bar{t})^{-1} B_{i}^{j}(\bar{t}) \bar{X}(\bar{t}) \\
& =\bar{X}(\bar{t})^{*} \mathbb{J} B_{i}^{j}(\bar{t}) \bar{X}(\bar{t}) .
\end{aligned}
$$

We conclude by (6). Since the matrix $S(T) S(\bar{t})^{-1}$ is invertible and (8) holds, we infer that

$$
\operatorname{Tr}\left(Y^{*} H\right)=0 \quad \forall H \in T_{\bar{M}(T)} \operatorname{Sp}(m),
$$

which yields a contradiction.

As a corollary, we deduce a local controllability property on $\operatorname{Sp}(m)$. We recall that $\operatorname{Sp}(m)$ has dimension $p:=2 m(2 m+1) / 2$.

Proposition 2.2. Assume that assumptions of Proposition 2.1 hold. Then, for every $\bar{X} \in S p(m)$ such that $\bar{u} \equiv 0$ belongs to $\mathcal{U}_{\bar{X}} \subset L^{1}\left([0, T] ; \mathbb{R}^{k}\right)$, there are $\mu, \nu>0$, $p$ smooth controls $u^{1}, \cdots, u^{p}:[0, T] \rightarrow \mathbb{R}^{k}$ with $\operatorname{Supp}\left(u^{j}\right) \subset(0, T)$ for $j=1, \ldots, p$ and a smooth mapping

$$
U=\left(U_{1}, \cdots, U_{p}\right): B(\bar{X}(T), \mu) \cap S p(m) \longrightarrow B(0, \nu)
$$

with $U(\bar{X}(T))=0$ such that

$$
E^{\bar{X}}\left(\sum_{j=1}^{p} U_{j}(X) u^{j}\right)=X \quad \forall X \in B(\bar{X}(T), \mu) \cap S p(m) .
$$

Proof. From Proposition 2.1, we know that the mapping $E^{\bar{X}}: \mathcal{U}_{\bar{X}} \rightarrow \operatorname{Sp}(m)$ is a smooth submersion at $\bar{u}$. Thus, remembering that the set of controls $u \in C^{\infty}\left([0, T] ; \mathbb{R}^{k}\right)$ with $\operatorname{Supp}(u) \subset(0, T)$ is dense in $L^{1}\left([0, T] ; \mathbb{R}^{k}\right)$, there are $p$ smooth controls $u^{1}, \ldots, u^{p}$ : $[0, T] \rightarrow \mathbb{R}^{k}$ with $\operatorname{Supp}\left(u^{j}\right) \subset(0, T)$ for $j=1, \ldots, p$ such that

$$
\operatorname{Span}\left\{d E^{\bar{X}}(\bar{u})\left(u^{j}\right) \mid j=1, \ldots, p\right\}=T_{\bar{X}(T)} \operatorname{Sp}(m) .
$$

Let $\Lambda>0$ be such that, for every $\lambda \in B(0, \Lambda)$, the control $\sum_{j=1}^{p} \lambda_{j} u^{j}$ belongs to $\mathcal{U}_{\bar{X}}$. Define $F: B(0, \Lambda) \rightarrow \mathrm{Sp}(m)$ by

$$
F(\lambda):=E^{\bar{X}}\left(\bar{u}+\sum_{j=1}^{p} \lambda_{j} u^{j}\right) \quad \forall \lambda=\left(\lambda_{1}, \ldots, \lambda_{p}\right) \in B(0, \Lambda) .
$$


The function $F$ is well-defined, smooth on $B(0, \Lambda)$, and satisfies $F(0)=E^{\bar{X}}(\bar{u})=$ $\bar{X}(T)$. Its differential at $\lambda=0$ is given by

$$
d F(0)(\lambda)=\sum_{j=1}^{p} \lambda_{j} d E^{\bar{X}}(\bar{u})\left(u^{j}\right) \quad \forall \lambda \in \mathbb{R}^{p},
$$

hence it is invertible by (12). By the Inverse Function Theorem, there are $\mu, \nu>0$ and a smooth function

$$
U=\left(U_{1}, \ldots, U_{p}\right): B(\bar{X}(T), \mu) \cap \operatorname{Sp}(m) \longrightarrow B(0, \nu)
$$

with $U(\bar{X}(T))=0$ such that

$$
E^{\bar{X}}\left(\bar{u}+\sum_{i=1}^{k} U_{i}(z) u^{i}\right)=z \quad \forall z \in B^{k}(\bar{X}(T), \mu) \cap \operatorname{Sp}(m) .
$$

This concludes the proof.

\section{Perturbations of the Hamiltonian by potentials and their effect OVER THE HAMILTONIAN FLOW}

Let $M$ be a $C^{\infty}$ compact manifold $M$ of dimension $n \geq 2$ and $H: T^{*} M \longrightarrow \mathbb{R}$ be a $C^{k}$ Tonelli Hamiltonian, $k \geq 2$. Let us consider the Hamiltonian flow $\phi_{t}$ : $H^{-1}(E) \longrightarrow H^{-1}$ in a regular energy level $H=E$, and let $\theta=(\gamma, \beta):[0, T] \rightarrow$ $H^{-1}(E)$ be a closed orbit of period $T$. Consider a section $\mathcal{S} \subset H^{-1}(E)$ transverse to $\theta([0, T])$ at $\theta(0)$ and denote by $\Phi: \mathcal{S} \rightarrow \mathcal{S}$ the Poincaré return map. Set $\hat{X}:=d \Phi$. It is a linear symplectic mapping from $T_{\theta(0)} \mathcal{S}$ onto itself. Any $C^{k}$ potential $U: M \rightarrow \mathbb{R}$ such that

$$
U(\gamma(t))=0, \quad d_{\gamma(t)} U=0 \quad \forall t \in[0, T],
$$

preserves the closed orbit $\theta$ and its energy level. If $U$ is small enough in a neighborhood of $\gamma([0, T])$, the Poincaré return map $\Phi_{U}: \mathcal{S} \rightarrow \mathcal{S}$ associated to the Hamiltonian flow of $H_{U}$ in $H_{U}^{-1}(E)$ and its differential $d \Phi_{U}: T_{\theta(0)} \mathcal{S} \rightarrow T_{\theta(0)} \mathcal{S}$ are well-defined. Our aim is to show that the set of $d \Phi_{U}$ for $U$ as above small enough contains an open subset of the set of linear symplectic matrices from $T_{\theta(0)} \mathcal{S}$ onto itself. We mention that in the construction that will be performed in the sequel, restricting to smaller interval $[a, b] \subset[0, T]$, we may always assume that

$$
\gamma(t) \cap \gamma([0, T] \backslash\{t\})=\emptyset \quad \forall t \in[a, b] .
$$


3.1. Good coordinates for the Hamiltonian flow along a non-singular orbit. The following result is proved in [12] (see [12, Lemma C.1]) after an application of a Lemma obtained by Li and Nirenberg (Lemma 3.1 in [14]). Let $\pi: T^{*} M \longrightarrow M$ be the canonical projection, let $\Psi: \mathcal{O} \subset M \longrightarrow B \subset \mathbb{R}^{n}$ be a local coordinate system for $M, \Psi(x)=\left(p_{1}(x), p_{2}(x), . ., p_{n}(x)\right)$, let $(p, q)$ be the canonical coordinates in $T^{*} M$ associated to $\Psi$, where $q=\Psi^{*} p$ represents the coordinates of 1 -forms induced by the dual forms $d p_{i}$ of the coordinate vector basis.

Lemma 3.1. Let $\theta([a, b])$ be a non-singular orbit of the Hamiltonian flow of $H$ without self-intersection. Let $x=\pi(\theta)$. Up to do a translation in the $q$ variable and to take a smaller interval, there exist local coordinates $\Phi: \mathcal{O} \subset M \longrightarrow \mathbb{R}^{n}$, $\Phi=\left(p_{1}, p_{2}, . ., p_{n}\right)$, where $\mathcal{O}$ is an open neighborhood of $x$ and $\Phi(x)=0$, such that the Hamiltonian $\bar{H}=\Phi_{*} H$ defined in an open set $\mathcal{V} \times \mathbb{R}^{n} \subset \mathbb{R}^{n} \times \mathbb{R}^{n}$ satisfies the following properties:

(1) The orbit of $\bar{H}$ through $\left(0, e_{1}\right)$ is $\bar{\phi}_{t}\left(0, e_{1}\right)=\left(t e_{1}, e_{1}\right)$ for every $t \in[a, b]$.

(2) In the coordinates $(p, q)$ the Hamiltonian satisfies

(i) $\frac{\partial^{2} \bar{H}}{\partial p_{i} \partial q_{j}}\left(\bar{\phi}_{t}(0)\right)=0$ for any $i, j=1, \ldots, n$.

(ii) $\frac{\partial^{2} \bar{H}}{\partial q_{1} \partial q_{j}}\left(\bar{\phi}_{t}(0)\right)=0$ for any $j=2, \ldots, n$.

(iii) The $(n-1) \times(n-1)$ matrix whose entries are $\frac{\partial^{2} \bar{H}}{\partial q_{i} \partial q_{j}}\left(\bar{\phi}_{t}(0)\right), 2 \leq i, j, \leq n$, is the identity matrix $I_{n-1}$.

Let us make some remarks about Lemma 3.1. It can be viewed as a generalization of well known special coordinate systems defined along Riemannian or Finslerian geodesics. Indeed, in the case of Riemannian geometry such coordinate system is given by Fermi coordinates (see [23] for instance), in the case of Finsler geometry the coordinate system arises from the existence of the Chern-Rund connection (see [4] for the definition), which is a covariant derivative defined along each Finslerian geodesic behaving just as a Riemannian connection. Notice that item (2) (ii) is a generalization of the notion of parallelism of the coordinate vector fields along the orbit $\bar{\phi}_{t}(\theta)$. In both (Riemannian and Finsler) cases, we can represent the differential of the geodesic flow in the unit tangent bundle along an orbit by a one parameter family of matrices $W(t)$ satisfying a first order system of the form:

$$
W^{\prime}(t)=\left(\begin{array}{cc}
0 & I \\
-K(t) & 0
\end{array}\right) W(t)
$$

The matrix $W(t)$ represents a basis of Jacobi fields and its derivatives defined in the orbit, and the matrix $K$ represents the sectional curvatures (flag curvatures in the 
Finsler case) of planes formed by the tangent vector of the geodesic associated to the orbit and the basis of the coordinate vector fields which are transverse to the geodesic. So Lemma 3.1 tells us that there exists a sort of Riemannian local form for the Hamiltonian flow in a neighborhood of a closed, non-singular geodesic, where the curvature matrix is replaced by the Hessian of the Hamiltonian with respect to these special coordinates. Moreover,

Lemma 3.2. If a property of $\bar{H}$ is Mañé $C^{k}$ generic, then the same holds for $H$.

This is just because there is a correspondence between perturbations of $\bar{H}$ by locally supported potentials and perturbations of $H$ by potentials that are locally supported in $U$.

3.2. Perturbations of the Hamiltonian by quadratic potentials. Applying Lemma 3.1 to a piece of a closed orbit $\theta$ for the Hamiltonian flow of $H$, we may assume that $\bar{\theta}=(\bar{p}, \bar{q})=\bar{\phi}_{t}\left(0, e_{1}\right):[a=0, b] \rightarrow \mathbb{R}^{n} \times \mathbb{R}^{n}$ is an orbit of a $C^{k}$ Tonelli Hamiltonian $\bar{H}: \mathbb{R}^{n} \times \mathbb{R}^{n} \rightarrow \mathbb{R}$ satisfying items (1)-(2) of Lemma 3.1. Thanks to Lemma 3.2, we just need to study generic perturbations of $\bar{H}$ in a neighborhood $\mathcal{V}$ of $\bar{p}([a, b])$.

Set $m:=n-1, \hat{p}:=\left(p_{2}, \ldots, p_{n}\right) \in \mathbb{R}^{m}$ for any $p=\left(p_{1}, \ldots, p_{n}\right) \in \mathbb{R}^{n}$, and fix $\delta>0$ such that $[a, b] \times B\left(0_{m}, \delta\right) \subset \mathcal{V}$. Pick two $C^{\infty}$ functions $c: \mathbb{R} \longrightarrow \mathbb{R}$ and $\tau: \mathbb{R}^{m} \rightarrow \mathbb{R}$ such that

$$
\begin{gathered}
c\left(t e_{1}\right)=\tau(\hat{p})=1 \quad \forall t \in[a, b], \forall \hat{p} \in B\left(0_{m}, \delta / 2\right), \\
\operatorname{Supp}(c) \times \operatorname{Supp}(\tau) \subset \mathcal{V},
\end{gathered}
$$

and define for every $i, j \in\{1, \ldots, m\}$ the $C^{\infty}$ function $\sigma_{i j}: \mathbb{R}^{n} \longrightarrow \mathbb{R}$ by

$$
\sigma_{i j}(p)=c\left(p_{1}\right) \tau(\hat{p}) p_{i+1} p_{j+1} \quad \forall p \in \mathbb{R}^{n} .
$$

For every $C^{\infty}$ function

with

$$
u=\left(u_{i j}\right)_{\substack{i, j=1, \ldots, m \\ i \leq j}}: \mathbb{R} \longrightarrow \mathbb{R}^{\frac{m(m+1)}{2}}
$$

$$
\operatorname{Supp}(u) \subset(a, b),
$$

we define the $C^{\infty}$ potential $U_{u}: \mathbb{R}^{n} \rightarrow \mathbb{R}$ with $\operatorname{Supp}(U) \subset \mathcal{V}$ by

$$
U_{u}(p):=\frac{1}{2} \sum_{i=1}^{m} u_{i i}\left(p_{1}\right) \sigma_{i i}(p)+\sum_{\substack{i, j=1 \\ i<j}}^{m} u_{i j}\left(p_{1}\right) \sigma_{i j}(p) \quad \forall p \in \mathbb{R}^{n} .
$$


Since the functions $\sigma_{i j}$ and their first derivatives vanish along the segment $\bar{p}([a, b])$, the trajectory $\bar{\theta}: t \in[a, b] \rightarrow\left(t e_{1}, e_{1}\right)$ is an orbit of the Hamiltonian flow of $\bar{H}_{u}$ given by

$$
\bar{H}_{u}(p, q)=\bar{H}(p, q)+U_{u}(p) \quad \forall(p, q) \in \mathbb{R}^{n} \times \mathbb{R}^{n} .
$$

Moreover, the $\bar{H}_{u}$-energy of the orbit of $\theta$ is the same $\bar{H}$-energy, and from Lemma 3.1 it is straightforward to check (using Hamilton equations) that the $2 m$-plane $\Pi \subset \mathbb{R}^{n} \times \mathbb{R}^{n}$ defined by

$$
\Pi=\left(\{0\} \times \mathbb{R}^{m}\right) \times\left(\{0\} \times \mathbb{R}^{m}\right)
$$

is invariant by the differential of the Hamiltonian flows of $\bar{H}, \bar{H}_{u}$ along $\bar{\theta}$.

Let $W_{u}:[a, b] \rightarrow M_{2 n}(\mathbb{R})$ be the differential of the Hamiltonian flow of $\bar{H}_{u}$ along $\bar{\theta}$. By Lemma 3.1 and the choice of the functions $u_{i j}$, the restriction $\hat{W}_{u}$ of $W_{u}$ to $\Pi$ satisfies the following first order Cauchy problem:

$$
\hat{W}_{u}^{\prime}(t)=\left(\begin{array}{cc}
0 & I_{m} \\
-K_{u}(t) & 0
\end{array}\right) \hat{W}_{u}(t), \quad \hat{W}_{u}(a)=I_{2 m}
$$

where $K_{u}(t)$ is defined from the matrix $K(t):=\left(\frac{\partial^{2} \bar{H}}{\partial p_{i} \partial p_{j}}\left(\bar{\phi}_{t}(0)\right)\right)_{i, j=2, \ldots, n}$, by the following formulae:

$$
K_{u}(t)=K(t)+\sum_{i=1}^{m} u_{i i}(t) E(i i)+\sum_{\substack{i, j=1 \\ i<j}}^{m} u_{i j}(t) E(i j),
$$

where the $E(i j), 2 \leq i \leq j \leq m$ are the symmetric $m \times m$ matrices defined by

$$
(E(i i))_{k, l}:=\delta_{i k} \delta_{i l} \quad \forall i=1, \ldots, m
$$

$$
\text { and } \quad(E(i j))_{k, l}=\delta_{i k} \delta_{j l}+\delta_{i l} \delta_{j k} \quad \forall i<j=1, \ldots, m \text {. }
$$

\section{Proof of Theorem 1.2}

Set $m=n-1, k:=m(m+1) / 2$ and for sake of simplicity assume from now that $[a, b]=[0, T]$. The formulas (13)-(14) giving $W_{u}(T)$ can be viewed as a control system of the form

$$
\dot{X}(t)=A(t) X(t)+\sum_{i \leq j=1}^{m} u_{i j}(t) \mathcal{E}(i j) X(t),
$$


where the $2 m \times 2 m$ matrices $A(t), \mathcal{E}(i j)$ are defined by

$$
A(t):=\left(\begin{array}{cc}
0 & I_{m} \\
-K(t) & 0
\end{array}\right) \quad \forall t \in[0, T]
$$

and

$$
\mathcal{E}(i j):=\left(\begin{array}{cc}
0 & 0 \\
E(i j) & 0
\end{array}\right),
$$

Since our control system has the form (1), all the results gathered in Section 2 apply. Since the $\mathcal{E}(i j)$ do not depend on time, we check easily that the matrices $B_{i j}^{0}, B_{i j}^{1}, B_{i j}^{2}, B_{i j}^{3}$ associated to our system are constant and given by

$$
\left\{\begin{array}{l}
B_{i j}^{0}(t)=\mathcal{E}(i j) \\
B_{i j}^{1}(t)=[\mathcal{E}(i j), A(t)] \\
B_{i j}^{2}(t)=[[\mathcal{E}(i j), A(t)], A(t)] \\
B_{i j}^{3}(t)=[[[\mathcal{E}(i j), A(t)], A(t)], A(t)],
\end{array}\right.
$$

for every $t \in[0, T]$. An easy computation yields for any $i, j=1, \ldots, m$ with $i \leq j$ and any $t \in[0, T]$,

$$
\begin{gathered}
{[\mathcal{E}(i j), A(t)]=\left(\begin{array}{cc}
-E(i j) & 0 \\
0 & E(i j)
\end{array}\right),} \\
{[[\mathcal{E}(i j), A(t)], A(t)]=\left(\begin{array}{cc}
0 & -2 E(i j) \\
-E(i j) K(t)-K(t) E(i j) & 0
\end{array}\right),} \\
{[[[\mathcal{E}(i j), A(t)], A(t)], A(t)]} \\
=\left(\begin{array}{cc}
3 E(i j) K(t)+K(t) E(i j) & 0 \\
0 & -E(i j) K(t)-3 K(t) E(i j)
\end{array}\right) .
\end{gathered}
$$

In order to apply Proposition 2.2, we need to check (8) for some $\bar{t} \in[0, T]$. The following result holds.

Lemma 4.1. Assume that there is $\bar{t} \in[0, T]$ such that

$$
\operatorname{dim}(\operatorname{Span}\{[E(i j), K(\bar{t})] \mid i, j \in\{1, \ldots, m\}, i<j\})=\frac{m(m-1)}{2} .
$$

Then

$$
\operatorname{Span}\left\{B_{i j}^{l}(\bar{t}) \mid i, j \in\{1, \ldots, m\}, i \leq j, l=0,1,2,3\right\}=T_{I_{2 m}} S p(m) .
$$


Proof. Since (6) holds, the following inclusion holds

$$
S:=\operatorname{Span}\left\{B_{i j}^{l}(\bar{t}) \mid i, j \in\{1, \ldots, m\}, i \leq j, l=0,1,2,3\right\} \subset T_{I_{2 m}} \operatorname{Sp}(m) .
$$

So, we just need to show that $S$ has dimension $p=2 m(2 m+1) / 2$. First, since the matrices $E(i j)$ form a basis of the vector space of symmetric matrices $\mathcal{S}(m)$, we check easily that the vector space

$$
\operatorname{Span}\left\{B_{i j}^{l}(\bar{t}) \mid i, j \in\{1, \ldots, m\}, i \leq j, l=0,2\right\}
$$

has dimension $m(m+1)$. Thanks to the block forms of the matrices $B_{i j}^{l}(\bar{t})$ with $l=0,1,2,3$, it remains to check that under assumption (16) the vector space

$$
\operatorname{Span}\left\{B_{i j}^{l}(\bar{t}) \mid i, j \in\{1, \ldots, m\}, i \leq j, l=1,3\right\}
$$

has dimension $p-m(m+1) / 2=m^{2}$. We claim that if (16) holds, the vector space

$$
\operatorname{Span}\left(\left\{B_{i j}^{1}(\bar{t}) \mid i, j \in\{1, \ldots, m\}, i \leq j\right\} \cup\left\{B_{i j}^{3}(\bar{t}) \mid i, j \in\{1, \ldots, m\}, i<j\right\}\right)
$$

has dimension $m^{2}$. Let us prove the claim arguing by contradiction. Set

$$
\mathcal{F}(i j):=B_{i j}^{3}(\bar{t})=\left(\begin{array}{cc}
3 E(i j) K(\bar{t})+K(\bar{t}) E(i j) & 0 \\
0 & -E(i j) K(\bar{t})-3 K(\bar{t}) E(i j)
\end{array}\right),
$$

for any $i, j \in\{1, \ldots, m\}$ with $i \leq j$ and assume that

$$
\sum_{\substack{i, j=1 \\ i<j}}^{m} a_{i j} \mathcal{F}(i j)+\sum_{\substack{i, j=1 \\ i \leq j}}^{m} b_{i j} B_{i j}^{1}(\bar{t})=0
$$

for some $a_{i j}, b_{i j}$. By the above formulas, we get

$$
\left\{\begin{array}{l}
\sum_{\substack{i, j=1 \\
i<j}}^{m} a_{i j}(3 E(i j) K(\bar{t})+K(\bar{t}) E(i j))+\sum_{\substack{i, j=1 \\
i \leq j}}^{m} b_{i j}(-E(i j))=0 \\
\sum_{\substack{i, j=1 \\
i<j}}^{m} a_{i j}(-E(i j) K(\bar{t})-3 K(\bar{t}) E(i j))+\sum_{\substack{i, j=1 \\
i \leq j}}^{m} b_{i j}(E(i j))=0 .
\end{array}\right.
$$

Summing up both lines yields

$$
\sum_{\substack{i, j=1 \\ i<j}}^{m} a_{i j}(2 E(i j) K(\bar{t})-2 K(\bar{t}) E(i j))=0
$$

which can be written as

$$
\sum_{\substack{i, j=1 \\ i<j}}^{m} a_{i j}[E(i j), K(\bar{t})]=0
$$


This contradicts assumption (16). Then we deduce that all the $a_{i j}$ 's vanish. Returning to (17) and using that the $E_{i j}$ are linearly independent, we conclude easily.

The result below follows easily from Proposition 2.2 and Lemma 4.1. We set $\bar{X}:=I_{2 m}$ and use the notations defined in Section 2 .

Proposition 4.2. Assume that there exists $\bar{t} \in[0, T]$ such that (16) holds. Then there are $\mu, C>0$ such that for every $X \in S p(m)$ with $\|X-\bar{X}(T)\|<\mu$, there is a $C^{\infty}$ function $u:[0, T] \rightarrow \mathbb{R}^{\frac{m(m+1)}{2}}$ such that

$$
\operatorname{Supp}(u) \subset(0, T), \quad\|u\|_{C^{k}}<C\|X-\bar{X}(T)\|
$$

and

$$
X_{u}(T)=X
$$

Proposition 4.2 shows that as soon as (16) holds for some $\bar{t} \in[0, T]$, the Hamiltonian can be perturbed by small potential in order to reach a small ball of symplectic matrices around $W_{0}(T)$. If (16) is never satisfied on $[0, T]$, we need first to add a small potential to $\bar{H}$ in order to satisfy (16) and then we apply Proposition 4.2. Such strategy is made possible by the following result.

Lemma 4.3. The set of matrices $K \in \mathcal{S}(m)$ such that

$$
\operatorname{dim}(\operatorname{Span}\{[E(i j), K] \mid i, j \in\{1, \ldots, m\}, i<j\})=\frac{m(m-1)}{2}
$$

is open and dense in $\mathcal{S}(m)$.

Proof. First, note that if $K, K^{\prime}$ belong to $\mathcal{S}(m)$ then $\left[K, K^{\prime}\right]$ is skew-symmetric. Denote by $\mathcal{S}^{-}(m)$ the set of $m \times m$ skew-symmetric matrices, it is a vector space of dimension $m(m-1) / 2$. Define the linear mapping

$$
\begin{aligned}
\Phi: \mathcal{S}(m) & \longrightarrow\left(\mathcal{S}^{-}(m)\right)^{\frac{m(m-1)}{2}} \\
K & \longmapsto\left(\left[E_{i j}, K\right]\right)_{\substack{i, j=1 \\
i<j}}
\end{aligned}
$$

We need to check that there is an open dense set of $K \in \mathcal{S}(m)$ such that the $m(m-1) / 2$ coordinates of $\Phi(K)$ are linearly independent in $\mathcal{S}^{-}(m)$ this amounts to show that the determinant of $\Phi(K)$ does not vanish identically in $\mathcal{S}(m)$. Define $\bar{K} \in \mathcal{S}(m)$ by

$$
\bar{K}_{r s}:=r \delta_{r s} \quad \forall r, s=1, \ldots, m .
$$

It is a diagonal matrix whose the coefficients are all distincts. We check easily that $\operatorname{det}(\Phi(K)) \neq 0$. Thus the set of symmetric matrices $K$ such that $\operatorname{det}(\Phi(K))$ is an 
algebraic submanifold of $\mathcal{S}(m)$ of dimension at most $m(m+1) / 2-1$. This shows that its complement is open and dense in $\mathcal{S}(m)$.

\section{Proof of the Kupka-Smale Theorem}

The proof of the Mañé's generic transversality of the intersection between invariant submanifolds of hyperbolic periodic orbits was proved by Oliveira [19]. Concerning the Mañé generic version of the closed orbits claimed in the Kupka-Smale theorem, Oliveira in [19] shows that it follows from the following statement (Theorem 18 in [19]):

Theorem 5.1. Let $H: T^{*} M \rightarrow \mathbb{R}$ be a Tonelli Hamiltonian, and let $\gamma \subset H^{-1}(E)$ be a periodic orbit contained in the regular energy level $H^{-1}(E)$ with minimal period $T$. Suppose that $\gamma$ is isolated in its energy level, non-degenerate of order $\leq m \in \mathbb{N}$. Then there exists a potential $U_{0} \in C^{\infty}(M, \mathbb{R})$ arbitrarily close to zero, with supp $\left(U_{0}\right) \subset$ $\mathcal{U} \subset M$ open, arbitrarily small, such that, $\gamma$ is nondegenerated of order $\leq 2 m$ to $H+U_{0}$.

We recall that a closed orbit $\gamma$ is non-degenerate of order $m \in \mathbb{N}$ if its minimum period is at most $m$ and no root of unity is an eigenvalue of the differential of the Poincaré map of $\gamma$. This theorem is proved in [19] in dimension 2.

The proof in the case of dimension $n$ follows from Proposition 4.2 and Lemma 4.3, where we showed that an open set of symplectic matrices is attained by a family of differentials of Poincaré maps of the closed orbit $\theta$ obtained by perturbing the initial Hamiltonian $H$ by adding small potentials. The set of matrices whose eigenvalues are either hyperbolic or unitary complex numbers whose imaginary part is irrational is dense. It is clear that if the Poincaré transform of a closed orbit has these properties, then the closed orbit will be $m$ non-degenerate for every $m$. This finishes the proof of Theorem 5.1 in any dimension.

\section{REFERENCES}

[1] R. Abrahams. Transversality in manifolds of mappings. Bull. Amer. Math. Soc., 69:470-474, 1963.

[2] R. Abrahams and J. Robbin. Transversal mappings and flows. Benjamin, New York, 1967.

[3] D.V. Anosov. Generic properties of closed geodesics. Izv. Akad. Nauk. SSSR Ser. Mat., 46(4):675-709, 896, 1982.

[4] D. Bao, S.-S. Chern and Z. Shen. An introduction to Riemann-Finsler geometry. Graduate Texts in Mathematics, vol. 200, Springer-Verlag, New York, 2000.

[5] P. Bernard and G. Contreras. A generic property of families of Lagrangian systems. Ann. of Math. (2), 167(3):1099-1108, 2008. 
[6] G. Contreras. Geodesic flows with positive topological entropy, twist maps and hyperbolicity. Ann. of Math. (2), 172(2):761-808, 2010.

[7] G. Contreras and R. Iturriaga. Convex Hamiltonians without conjugate points. Ergodic Theory Dynam. Systems, 19(4):901-952, 1999.

[8] G. Contreras and G.P. Paternain. Connecting orbits between static classes for generic Lagrangian systems. Topology, 41 (4):645-666, 2002.

[9] J.-M. Coron. Control and nonlinearity. Mathematical Surveys and Monographs, 136. American Mathematical Society, Providence, RI, 2007.

[10] R. Devaney. Reversible diffeomorphisms and flows. Trans. Amer. Math. Soc., 218:89-113, 1976.

[11] A. Figalli and L. Rifford. Closing Aubry sets I. Preprint, 2010.

[12] A. Figalli and L. Rifford. Closing Aubry sets II. Preprint, 2010.

[13] W. Klingenberg and F. Takens. Generic properties of geodesic flows. Math. Ann., 197:323-334, 1972.

[14] Y. Li and L. Nirenberg. The distance function to the boundary, Finsler geometry, and the singular set of viscosity solutions of some Hamilton-Jacobi equations. Comm. Pure Appl. Math., 58(1):85-146, 2005.

[15] R.Mañé, R.: Generic properties and problems of minimizing measures of Lagrangian systems. Nonlinearity 9 (1996) n. 2, 273-310.

[16] D. Massart. On Aubry sets and Mather's action functional. Israel J. Math., 134:157-171, 2003.

[17] J. Mather. Action minimizing measures for positive definite Lagrangian systems. Math. Z., 20(2):169-207, 1991.

[18] J. Moser, C. L. Siegel. Lectures on celestial mechanics. Springer Verlag, Berlin - Heildelberg, ISBN 9783540586562.

[19] E. Oliveira. Generic properties of Lagrangians on surfaces: the Kupka-Smale theorem. Discrete Contin. Dyn. Syst., 21(2):551-569, 2008.

[20] L. Rifford. Closing geodesics in $C^{1}$ topology. Preprint, 2010.

[21] C. Robinson. Generic properties of conservative systems I. Amer. J. Math., 92:562-603, 1970.

[22] C. Robinson. Generic properties of conservative systems II. Amer. J. Math., 92:897-906, 1970.

[23] R. Ruggiero. On the density of mechanical Lagrangians in $T^{2}$ without continuous invariant graphs in all supercritical energy levels. Discrete Contin. Dyn. Syst. Ser. B, 10(2-3):661-679, 2008.

\author{
LudOVIC RIFFORD
}

Université DE NiCE-Sophia Antipolis

Labo. J.-A. Dieudonné, UMR 6621

Parc Valrose, 06108 Nice Cedex 02

FRANCE

EMAIL: rifford@unice.fr

Rafael O. RugGiero 
PuC-Rio, Departamento de Matemática. Rua Marqués de São Vicente 225, Gávea, 22450-150, Rio de Janeiro, Brazil

EMAIL: rorr@mat .puc-rio.br 